\title{
The momentum operators corresponding to a localized massless particle
}

\author{
A.R. Assar and V. Putz ${ }^{1}$, \\ ${ }^{1}$ Institut für Photonik, Technische Universität Wien \\ Gusshausstr. 25-29, A-1040 Vienna, Austria \\ putz@hep.itp.tuwien.ac.at
}

November 13, 2018

\begin{abstract}
In this article we propose, using a purely group theoretical argument, that if a massless particle is localized, then there are only two momentum operator s corresponding to the localized state. We explicitly determine these self-adjoint operators.
\end{abstract}

PACS-1996: 02.20.Fh, 03.65.Fd

There have been suggestions [1] that a massless particle can be localized in the hyperplane perpendicular to the light-like momentum 4-vector of that particle. In what follows, we shall use a group theoretical method based on Mackey's induction-reduction theorem [2, 3], to obtain the momentum operators corresponding to such a localized state.

By the relativistic mass-momentum relation,

$$
p^{2}=p_{\mu} p^{\mu}=m^{2},
$$

if $m=0$, then $p^{2}=0$, and one may without loss of generality take the light-like vector $p$ to be

$$
p=\left(\begin{array}{l}
1 \\
0 \\
0 \\
1
\end{array}\right) .
$$

There is also a representation of any 4 -vector by $2 \times 2$ matrices as follows

$$
P=\left(\begin{array}{cc}
p_{0}+p_{3} & p_{1}+i p_{2} \\
p_{1}-i p_{2} & p_{0}-p_{3}
\end{array}\right)
$$


Therefore, the matrix $P$ corresponding to the 4 -vector $(\mathbb{I})$ is of the form

$$
P=\left(\begin{array}{ll}
2 & 0 \\
0 & 0
\end{array}\right) .
$$

The little group (or isotropy group) of this 4 -vector is the set of all matrices $A$ such that

$$
A \in S L(2, \mathbb{C}): A P A^{+}=P .
$$

Taking

$$
A=\left(\begin{array}{ll}
a & b \\
c & d
\end{array}\right)
$$

it follows from (3) that

$$
\left(\begin{array}{ll}
a & b \\
c & d
\end{array}\right)\left(\begin{array}{ll}
2 & 0 \\
0 & 0
\end{array}\right)\left(\begin{array}{cc}
\bar{a} & \bar{c} \\
\bar{b} & \bar{d}
\end{array}\right)=\left(\begin{array}{ll}
2 & 0 \\
0 & 0
\end{array}\right)
$$

or

$$
\left(\begin{array}{cc}
2|a|^{2} & 2 a \bar{c} \\
2 c \bar{a} & 2|c|^{2}
\end{array}\right)=\left(\begin{array}{cc}
2 & 0 \\
0 & 0
\end{array}\right) \Rightarrow|a|^{2}=1, \quad c=0 .
$$

Thus, the isotropy group of $P$ is the set of all matrices in $S L(2, \mathbb{C})$ of the form

$$
I S(P)=\left\{\left(\begin{array}{cc}
e^{i \theta} & b \\
0 & e^{-i \theta}
\end{array}\right) \mid b \in \mathbb{C}\right\} .
$$

It is clear that this is a subgroup of $S L(2, \mathbb{C})$ which is isomorphic to the Euclidean group

$$
E_{2}=T_{2} \rtimes S O(2) .
$$

The $T_{2}$-subgroup is clearly the set of matrices of the form

$$
T_{2}=\left\{\left(\begin{array}{ll}
1 & b \\
0 & 1
\end{array}\right) \mid b \in \mathbb{C}\right\} .
$$

The Euclidean $E_{2}$ is the group of Euclidean motion in a hyperplane orthogonal to the 4 -vector (10), and the $T_{2}$ subgroup corresponds to translations in this hyperplane.

By Wigner's argument particles are classified according to their mass and spin by the unitary irreducible representations of the Poincaré group, and that any such representation can be induced from 1-dimensional representations (or characters) of the little group corresponding to the momentum 4-vector of the particle in question. The Poincaré group is a semi-direct product

$$
\mathcal{P}=T_{4} \rtimes S L(2, \mathbb{C}),
$$


where $S L(2, \mathbb{C})$ is the double covering of the restricted Lorentz group $L_{0}$. It is this $S L(2, \mathbb{C})$-part, i.e., the Lorentz group part of the Poincaré group which concerns us in what follows. Consider the following two subgroups of $S L(2, \mathbb{C})$,

$$
\begin{aligned}
& H_{1}=\left\{\left(\begin{array}{cc}
\lambda & \mu \\
0 & \lambda^{-1}
\end{array}\right) \mid \lambda, \mu \in \mathbb{C}\right\}, \\
& H_{2}=\left\{\left(\begin{array}{ll}
1 & \beta \\
0 & 1
\end{array}\right) \mid \beta \in \mathbb{C}\right\} .
\end{aligned}
$$

Notice that $I S(P)$ is a subgroup of $H_{1}$. Also $H_{1}$ is the inducing subgroup for all principal series unitary IRR of $S L(2, \mathbb{C})$, 4] . Our aim is to find the reduction of a unitary representation of $S L(2, \mathbb{C})$ induced from a character of $H_{1}$, say $\Delta\left(H_{1}\right)$, when restricted to to $H_{2}$. This is given by Mackey's induction-reduction theorem in the form

$$
\left(\Delta\left(H_{1}\right) \uparrow S L(2, \mathbb{C})\right) \downarrow H_{2}=\sum_{\oplus d} \Delta_{d}\left(S_{d}\right) \uparrow H_{2},
$$

where the direct sum is over all the $H_{1} d H_{2}$ double cosets in $S L(2, \mathbb{C})$ of non-zero measure. This reduction is found in the following steps.

Step 1. The double cosets $H_{1} d H_{2}$ are found as follows. Naimark has shown [4] that the right coset representatives of $H_{1}$ in $S L(2, \mathbb{C})$ can be taken to be of the form

$$
Z=\left(\begin{array}{ll}
1 & 0 \\
z & 1
\end{array}\right), \quad z=x+i y \in \mathbb{C}
$$

The double coset representatives can be computed by

$$
\begin{aligned}
& \left(\begin{array}{cc}
1 & 0 \\
z^{\prime} & 1
\end{array}\right)\left(\begin{array}{cc}
1 & \beta \\
0 & 1
\end{array}\right)=\left(\begin{array}{cc}
\lambda & \mu \\
0 & \lambda^{-1}
\end{array}\right)\left(\begin{array}{cc}
1 & 0 \\
z & 1
\end{array}\right) \\
& \Rightarrow\left(\begin{array}{cc}
1-\beta z & \beta \\
z^{\prime}-z-\beta z z^{\prime} & \beta z^{\prime}+1
\end{array}\right)=\left(\begin{array}{cc}
\lambda & \mu \\
0 & \lambda^{-1}
\end{array}\right) .
\end{aligned}
$$

From the bottom left element of the matrix we find

$$
z^{\prime}=\frac{z}{1-\beta z} .
$$

If $z^{\prime}=0$, then $z=0$. This means that the origin in the complex plane $\mathbb{C}$ is a double coset itself. This corresponds to $d=\left(\begin{array}{ll}1 & 0 \\ 0 & 1\end{array}\right)$.

Next, suppose $z^{\prime}=1$. It follows from (9) that

$$
\beta=\frac{1}{z}-1,
$$

which shows that for every $\beta \in \mathbb{C}$ there exists a curve passing through $z^{\prime}=1$ and joining $z$ and $\beta$. Thus $\mathbb{C} \backslash\{0\}$ is another double coset, a representative of which may be taken to be any matrix of the form $\left(\begin{array}{cc}1 & 0 \\ z & 1\end{array}\right), z \neq 0$. 
We may choose $z=1$ and thus we have the following double coset decomposition

$$
S L(2, \mathbb{C})=H_{1}\left(\begin{array}{ll}
1 & 0 \\
0 & 1
\end{array}\right) H_{2} \cup H_{1}\left(\begin{array}{ll}
1 & 0 \\
1 & 1
\end{array}\right) H_{2} .
$$

However, the double coset $H_{1}\left(\begin{array}{ll}1 & 0 \\ 0 & 1\end{array}\right) H_{2}$, corresponding to the origin, has measure zero. Thus there is only one double coset of non-zero measure, i.e.,

$$
S L(2, \mathbb{C})=H_{1}\left(\begin{array}{ll}
1 & 0 \\
1 & 1
\end{array}\right) H_{2}, \quad \text { almost everywhere. }
$$

Step 2. Next, consider the subgroup

$$
S_{d}=H_{1} \cap d^{-1} H_{2} d,
$$

where $d=\left(\begin{array}{ll}1 & 0 \\ 1 & 1\end{array}\right)$.

$$
\begin{aligned}
d^{-1} H_{2} d= & \left(\begin{array}{cc}
1 & 0 \\
-1 & 1
\end{array}\right)\left(\begin{array}{ll}
1 & \beta \\
0 & 1
\end{array}\right)\left(\begin{array}{ll}
1 & 0 \\
1 & 1
\end{array}\right)=\left(\begin{array}{cc}
1+\beta & \beta \\
-\beta & 1-\beta
\end{array}\right), \\
S_{d} & =\left(\begin{array}{cc}
\lambda & \mu \\
0 & \lambda^{-1}
\end{array}\right) \cap\left(\begin{array}{cc}
1+\beta & \beta \\
-\beta & 1-\beta
\end{array}\right)=\left(\begin{array}{ll}
1 & 0 \\
0 & 1
\end{array}\right)=\{e\},
\end{aligned}
$$

which is just the identity element. Thus $S_{d}$ is the trivial subgroup of $S L(2, \mathbb{C})$.

By Mackey's theorem, we have

$$
\left(\Delta\left(H_{1}\right) \uparrow S L(2, \mathbb{C})\right) \downarrow H_{2}=\mathbf{1}(e) \uparrow H_{2},
$$

where $\mathbf{1}(e)$ is the 1-dimensional representation of the identity subgroup. Clearly, the right-hand side of (10) is just the regular representation of $H_{2}$.

Step 3. The quasi-invariant measure on $H_{1} \backslash S L(2, \mathbb{C})$. As mentioned earlier the right coset representatives of $H_{1}$ in $S L(2, \mathbb{C})$ may be taken to be of the form (8). Let

$$
g=\left(\begin{array}{ll}
g_{11} & g_{12} \\
g_{21} & g_{22}
\end{array}\right) \in S L(2, \mathbb{C}), \quad Z^{\prime}=\left(\begin{array}{cc}
1 & 0 \\
z^{\prime} & 1
\end{array}\right)
$$

be such that

$$
Z g \in H_{1} Z^{\prime} \quad \Rightarrow \quad Z g=h Z^{\prime}
$$

for some $h \in H_{1}$. Thus we have

$$
\begin{aligned}
\left(\begin{array}{cc}
1 & 0 \\
z & 1
\end{array}\right)\left(\begin{array}{cc}
g_{11} & g_{12} \\
g_{21} & g_{22}
\end{array}\right) & =\left(\begin{array}{cc}
\lambda & \mu \\
0 & \lambda^{-1}
\end{array}\right)\left(\begin{array}{cc}
1 & 0 \\
z^{\prime} & 1
\end{array}\right) \\
\left(\begin{array}{cc}
g_{11} & g_{12} \\
z g_{21}+g_{22} & z g_{12}+g_{22}
\end{array}\right) & =\left(\begin{array}{cc}
\lambda+\mu z^{\prime} & \mu \\
\lambda^{-1} z^{\prime} & \lambda^{-1}
\end{array}\right) \\
z^{\prime}=\frac{\lambda^{-1} z^{\prime}}{\lambda^{-1}} & =\frac{z g_{21}+g_{22}}{z g_{12}+g_{22}}
\end{aligned}
$$


Putting $z=x+i y, z^{\prime}=x^{\prime}+i y^{\prime}$ in (12) and separating the real and imaginary parts, one obtains

$$
d x^{\prime} d y^{\prime}=\left|z g_{12}+g_{22}\right|^{-4} d x d y .
$$

Hence, the corresponding $\lambda_{\rho}$-function $[3]$ is

$$
\lambda_{\rho}\left(H_{1} z, g\right)=\left|z g_{12}+g_{22}\right|^{-4} .
$$

Step 4. The quasi-invariant measure on $H_{2} / S_{d}$. The $\lambda_{\rho}$-function corresponding to the quasi-invariant measure on $H_{2} / S_{d}$ is

$$
\lambda_{\rho}\left(S_{d} Z^{\prime}, g\right)=\lambda_{\rho}\left(H_{1}\left(d Z^{\prime}\right), g\right),
$$

where $Z^{\prime}=\left(\begin{array}{cc}1 & z^{\prime} \\ 0 & 1\end{array}\right) \in H_{2}$ and $g \in H_{2}$.

$$
\begin{aligned}
& d Z^{\prime}=\left(\begin{array}{cc}
1 & 0 \\
1 & 1
\end{array}\right)\left(\begin{array}{cc}
1 & z^{\prime} \\
0 & 1
\end{array}\right)=\left(\begin{array}{cc}
1 & z^{\prime} \\
1 & 1+z^{\prime}
\end{array}\right)=k, \\
& \lambda_{\rho}\left(H_{1}\left(d Z^{\prime}\right), g\right)=\lambda_{\rho}\left(H_{1} k, g\right), \quad g \in H_{2} .
\end{aligned}
$$

We may decompose $k$ as

$$
\begin{aligned}
& k=h_{1} Z, \quad h_{1} \in H_{1}, \quad Z=\left(\begin{array}{cc}
1 & 0 \\
z & 1
\end{array}\right): \\
& \left(\begin{array}{cc}
1 & z^{\prime} \\
1 & 1+z^{\prime}
\end{array}\right)=\left(\begin{array}{cc}
\lambda & \mu \\
0 & \lambda^{-1}
\end{array}\right)\left(\begin{array}{cc}
1 & 0 \\
z & 1
\end{array}\right)=\left(\begin{array}{cc}
\lambda+\mu z & \mu \\
\lambda^{-1} z & \lambda^{-1}
\end{array}\right) \\
& \Rightarrow z=\frac{\lambda^{-1} z}{\lambda^{-1}}=\frac{1}{1+z^{\prime}} .
\end{aligned}
$$

Taking $g=\left(\begin{array}{cc}1 & \beta \\ 0 & 1\end{array}\right) \in H_{2}$, i.e. $g_{12}=\beta, g_{22}=1$, we have, using (14), that

$$
\lambda_{\rho}\left(H_{1} k, g\right)=\left|\frac{\beta}{1+z^{\prime}}+1\right|^{-4},
$$

and

$$
d x^{\prime} d y^{\prime}=\left|\frac{1+z^{\prime}+\beta}{1+z^{\prime}}\right|^{-4} d x d y
$$

is the required quasi-invariant measure on $H_{2} / S_{d}$.

Step 5. It follows that the unitary representation in (17) is given by

$$
U(g) f\left(Z^{\prime}\right)=\sqrt{\left|\frac{1+z^{\prime}+\beta}{1+z^{\prime}}\right|^{-4}} f\left(Z^{\prime} g\right), \quad Z^{\prime}, g \in H_{2}, \quad g=\left(\begin{array}{cc}
1 & \beta \\
0 & 1
\end{array}\right) .
$$


Using

$$
\left(\begin{array}{cc}
1 & z^{\prime} \\
0 & 1
\end{array}\right)\left(\begin{array}{ll}
1 & \beta \\
0 & 1
\end{array}\right)=\left(\begin{array}{cc}
1 & z^{\prime}+\beta \\
0 & 1
\end{array}\right),
$$

identifying

$$
\left(\begin{array}{cc}
1 & z^{\prime} \\
0 & 1
\end{array}\right) \leftrightarrow z^{\prime}, \quad \text { etc. }
$$

and finally changing $z^{\prime}$ to $z$, we obtain

$$
U(\beta) f(z)=\left|\frac{1+z}{1+z+\beta}\right|^{2} f(z+\beta) .
$$

This is a unitary representation of $H_{2}$, obtained by the restriction of a representation $\Delta\left(H_{1}\right) \uparrow S L(2,(C))$ to the subgroup $H_{2}$.

Step 6. Determination of the infinitesimal operators (i.e. the generalized Lie derivatives). The generators of $H_{2}$ are

$$
\begin{aligned}
& b_{1}=\left.\frac{d}{d \beta_{1}}\left(\begin{array}{cc}
1 & \beta_{1}+i \beta_{2} \\
0 & 1
\end{array}\right)\right|_{\beta=0}=\left(\begin{array}{ll}
0 & 1 \\
0 & 0
\end{array}\right), \\
& b_{2}=\left.\frac{d}{d \beta_{2}}\left(\begin{array}{cc}
1 & \beta_{1}+i \beta_{2} \\
0 & 1
\end{array}\right)\right|_{\beta=0}=\left(\begin{array}{ll}
0 & i \\
0 & 0
\end{array}\right) .
\end{aligned}
$$

The corresponding 1-parameter subgroups are

$$
g_{1}=\exp \left\{\beta_{1} b_{1}\right\}, \quad g_{2}=\exp \left\{\beta_{2} b_{2}\right\} .
$$

The associated infinitesimal operators are given by the Lie derivatives

$$
B_{i}=\lim _{\eta \rightarrow 0} \frac{U\left(\exp \left(\eta b_{i}\right)\right)-U(e)}{\eta}, \quad i=1,2 .
$$

That is

$$
\begin{aligned}
& B_{1} f(z)=\lim _{\beta_{1} \rightarrow 0} \frac{U\left(\beta_{1}\right) f(z)-U(0) f(z)}{\beta_{1}}, \\
& B_{2} f(z)=\lim _{\beta_{2} \rightarrow 0} \frac{U\left(i \beta_{2}\right) f(z)-U(0) f(z)}{\beta_{2}} .
\end{aligned}
$$

We compute $B_{1}$. Writing $f(z)=f(x, y)$, where $z=x+i y$, we have

$$
\begin{aligned}
& U\left(\beta_{1}\right) f(z)=U\left(\beta_{1}\right) f(x, y)=\left|\frac{z+1}{1+z+\beta_{1}}\right|^{2} f\left(x+\beta_{1}, y\right) \\
& U(0) f(z)=f(z)=f(x, y) \\
& B_{1} f(z)=\lim _{\beta_{1} \rightarrow 0} \frac{|z+1|^{2} \frac{f\left(x+\beta_{1}, y\right)}{\left|1+z+\beta_{1}\right|^{2}}-f(x, y)}{\beta_{1}}
\end{aligned}
$$


Let

$$
\frac{f\left(x+\beta_{1}, y\right)}{\left|1+z+\beta_{1}\right|^{2}}=\frac{f\left(x+\beta_{1}, y\right)}{\left(1+x+\beta_{1}\right)^{2}+y^{2}}=: F(x+\beta, y) .
$$

Expanding this function in a Taylor series

$$
\begin{aligned}
& F(x+\beta, y)=F(x, y)+\left.\beta_{1} \frac{\partial}{\partial x} F\left(x+\beta_{1}, y\right)\right|_{\beta_{1}=0}+\ldots \\
& =\frac{f(x, y)}{|z+1|^{2}} \\
& +\left.\beta_{1} \frac{\left|z+1+\beta_{1}\right|^{2} \frac{\partial}{\partial x} f\left(x+\beta_{1}, y\right)-2\left(x+1+\beta_{1}\right) f\left(x+\beta_{1}, y\right)}{\left|z+\beta_{1}+1\right|^{4}}\right|_{\beta_{1}=0}+\ldots
\end{aligned}
$$

Replacing this in (19) we obtain

$$
B_{1} f(z)=\left\{\frac{-2(x+1)}{|z+1|^{2}}+\frac{\partial}{\partial x}\right\} f(z),
$$

and hence we have

$$
B_{1}=\frac{-2(x+1)}{(x+1)^{2}+y^{2}}+\frac{\partial}{\partial x} .
$$

A similar computation yields

$$
B_{2}=\frac{-2 y}{(x+1)^{2}+y^{2}}+\frac{\partial}{\partial y} .
$$

Applying a change of variable, $x+1 \rightarrow x$, one obtains a more symmetric form,

$$
B_{1}=\frac{-2 x}{x^{2}+y^{2}}+\frac{\partial}{\partial x}, \quad B_{2}=\frac{-2 y}{x^{2}+y^{2}}+\frac{\partial}{\partial y} .
$$

Note that $\left[B_{1}, B_{2}\right]=0$ as one expects.

Step 7. The measure which makes the space of functions $f(z)$ in (18) into a Hilbert space $L^{2}(-\infty,+\infty)$ is the same measure with respect to which the representation $U(\beta)$ is unitary,

$$
\left(U(\beta) f_{1}(z), U(\beta) f_{2}(z)\right)=\left(f_{1}(z), f_{2}(z)\right) .
$$

This measure is obtained from relation (15),

$$
\begin{aligned}
& z=\frac{1}{1+z^{\prime}}=\frac{\left(1+x^{\prime}\right)-i y^{\prime}}{\left(1+x^{\prime}\right)^{2}+y^{\prime 2}} \Rightarrow \\
& x=\frac{1+x^{\prime}}{\left(1+x^{\prime}\right)^{2}+y^{\prime 2}}, \quad y=\frac{-y^{\prime}}{\left(1+x^{\prime}\right)^{2}+y^{\prime 2}} \\
& d x d y=\left|\begin{array}{cc}
\frac{\partial x}{\partial x^{\prime}} & \frac{\partial y}{\partial x^{\prime}} \\
\frac{\partial x}{\partial y^{\prime}} & \frac{\partial y}{\partial y^{\prime}}
\end{array}\right| d x^{\prime} d y^{\prime}=\frac{d x^{\prime} d y^{\prime}}{\left|1+z^{\prime}\right|^{4}},
\end{aligned}
$$


and if the same change of variables, $x+1 \rightarrow x, y \rightarrow y$ is applied, one obtains

$$
d x d y=\frac{1}{\left|z^{\prime}\right|^{4}} d x^{\prime} d y^{\prime}=\frac{d x^{\prime} d y^{\prime}}{\left(x^{\prime 2}+y^{\prime 2}\right)^{2}} .
$$

The $L^{2}(-\infty,+\infty)$ space of functions $f(z)$ has a product given by

$$
\left(f_{1}(z), f_{2}(z)\right)=\int_{-\infty}^{+\infty} \int_{-\infty}^{+\infty} \overline{f_{1}(z)} f_{2}(z) \frac{d x d y}{|z|^{4}} .
$$

It is easily seen that this is the product with respect to which $U(\beta)$ is unitary.

With proper behaviour of $f_{1}, f_{2}$ at infinity, the operators $B_{1}$ and $B_{2}$ given by (22) are antisymmetric,

$$
\left(B_{i} f, g\right)=-\left(f, B_{i} g\right), \quad i=1,2 \text {. }
$$

Therefore, the operators

$$
\begin{aligned}
& \Pi_{1}=i B_{1}=-\frac{2 i x}{x^{2}+y^{2}}+i \frac{\partial}{\partial x} \\
& \Pi_{2}=i B_{2}=-\frac{2 i y}{x^{2}+y^{2}}+i \frac{\partial}{\partial y}
\end{aligned}
$$

are self-adjoint and their commutator vanishes. As these operators correspond to translations in the hyperplane introduced earlier, they are the momentum operators acting on the wave function of the localized massless particle.

Introducing the operator

$$
P^{2}:=\Pi_{1}^{2}+\Pi_{2}^{2}
$$

and using the fact that $\left[P^{2}, \Pi_{1}\right]=0=\left[P^{2}, \Pi_{2}\right]$, the simultaneous eigenfunctions of $\Pi_{1}$ and $\Pi_{2}$ can be computed from

$$
P^{2} \varphi=\lambda^{2} \varphi
$$

The computation of $\varphi$ is easily done by writing the expression for $P^{2}$ in polar coordinates corresponding to the cartesian coordinates $(x, y)$. W e propose that the wave function corresponding to the localized massless state is a superposition of all such eigenstates.

\section{References}

[1] A.O. Bahut, R. Raczka, Theory of group representations and applications, PWN-Polish scientific publishers, 1980.

[2] G.W. Mackey, Ann. of Math 55 (1952), 101-139. Ann. of Math 58 (1953), 193-221. 
[3] A.-R. Assar, On Mackey's subgroup theorem, Report on Mathematical Physics No. 1, Vol. 24 (1986), pp. 57-64.

[4] M.A. Naimark, Linear representations of Lorentz group, New York, Macmillan, 1964. 